However, the corrosive nature of aqueous amines, coupled with the fact that they require heating to liberate the $\mathrm{CO}_{2}$ afterwards, means that these processes also come with an associated environmental cost.

Now, in an effort to develop alternative, lightweight materials for ambienttemperature carbon capture and release, James Tour and a team of researchers from Rice University and the National Institute of Standards and Technology in the USA have developed cheap carbonaceous materials to act as $\mathrm{CO}_{2}$ sorbents in natural-gas wells. Commercially available poly((2-hydroxymethyl)thiophene) or poly(acrylonitrile) were ground together with potassium hydroxide and then carbonized by heating to $600{ }^{\circ} \mathrm{C}$. This process produced solid porous carbon materials containing either sulfur or nitrogen atoms distributed throughout the framework. At room temperature and at pressures comparable to the partial pressure of $\mathrm{CO}_{2}$ in a natural gas well, it was found that the materials possess remarkably high $\mathrm{CO}_{2}$ uptake capacities relative to the proportion of heteroatoms - the expected sorption sites in the structure.

This high uptake was rationalized by spectroscopic evidence for the presence of polymeric $\mathrm{CO}_{2}$ in the pores of the material. The surprising formation of poly $\left(\mathrm{CO}_{2}\right)$ means that the sorption capacity is limited not by the number of heteroatoms, but by their available pore volume. Depressurization of the system results in the release of gaseous $\mathrm{CO}_{2}$, which could, in principle, be directed back into the ground at the gas well site. Given that the materials also display reasonable selectivity for sorption of $\mathrm{CO}_{2}$ over methane, assisted by the displacement of physisorbed gas by the nascent poly $\left(\mathrm{CO}_{2}\right)$ chains, they could represent cheap and accessible alternatives to aqueous amines for $\mathrm{CO}_{2}$ scrubbing.

\section{ANTICANCER AGENTS}

\section{Sensitive after a knockdown}

Angew. Chem. Int. Ed. http://dx.doi.org/10.1002/ anie.201405169 (2014)

Protein-protein interactions are extremely difficult to target with small molecules because of the lack of a defined pocket in which they can tightly bind. Nevertheless, protein-protein interactions are involved in many important cellular processes and so they are important therapeutic targets. One such target is the so-called BRCT domain of the tumour suppressor protein BRCA1. Mutations in BRCA1 have been found in around $50 \%$ of hereditary breast cancers, and the protein plays a key role in

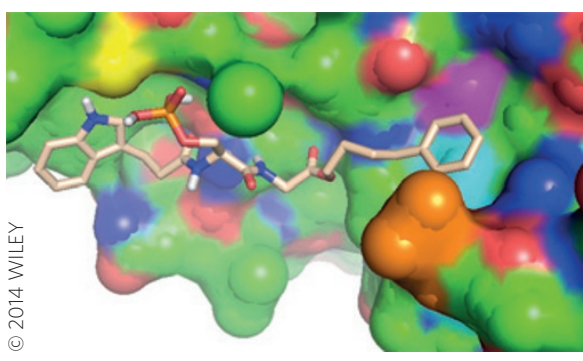

the response to DNA damage. Until now, the only reported examples of BRCA1 inhibitors were phosphopeptides, which need to be attached to cell-penetrating peptides to enable them to be taken up by tumour cells.

In an effort to develop the first cellpermeable, small-molecule inhibitor of BRCA1, Shao Yao and co-workers at the National University of Singapore have used a microarray-based screening approach. Phosphoserines are known to bind to BRCT domains, so the team started by screening a range of phosphopeptides to identify a preferred binding motif. This lead structure was then converted into a small molecule-like compound by screening a small-molecule microarray in which the peptides either side of the phosphoserine residue had been replaced with aliphatic and aromatic building blocks. Further computational refinement and in vitro screening suggested a promising candidate; however, a stability assay showed that it was rapidly degraded in mammalian cell lysates. To improve the stability, Yao and co-workers developed a non-hydrolysable analogue by switching the phosphoserine for difluoromethylenephosphoserine. A pro-drug version of this compound - with a pivaloxymethyl group attached to the phosphonate - was also developed in the hope that it would improve cell permeability.

The next step was to test the biological effectiveness of these compounds. Pull-down experiments showed that these compounds were potent binders of BRCA1 and were capable of disrupting a BRCA1 proteinprotein interaction in a dose-dependent manner. Encouraged by this, the team then investigated whether these compounds could be used to improve the effectiveness of anticancer treatments. Administration of the pro-drug sensitized a cancer-cell line to ionizing-radiation-induced apoptosis. In addition, it also showed a synergistic inhibitory effect on tumour cells when used in combination with the anticancer drugs Olaparib and Etoposide.

Written by Stuart Cantrill, Stephen Davey, Claire Hansell and Russell Johnson.

\section{blog $_{\text {roll }}$ 空}

\section{Yoghurt vs Scientists}

Recent advertising strategies in which 'antiscience' is equated with 'all-natural' have rubbed some scientists the wrong way.

Yoghurt maker Chobani earned the ire of the scientific community with a slogan that appeared on the lids of their new low-calorie Greek yoghurt. Piper Klemm was the first to tweet about the controversial catchphrase: 'Nature got us to 100 calories, not scientists. \#howmatters'. Hundreds of tweets on the topic soon followed, as well as numerous blog posts by scientists decrying the belittling of science in advertising.

Writing at In the Pipeline, Derek Lowe offered a tongue-in-cheek analysis (http:// go.nature.com/nwH2Ei) of the reason for the overwhelming response to the slogan, pointing out that despite Chobani's desire to advertise an 'all-natural' product, mass-produced yoghurt requires a lot of food science. In fact, Chad Jones and John Coupland describe the chemistry involved in detail in a podcast (http://go.nature.com/ $\mathrm{Kep}(\mathrm{HC}$ ) at The Collapsed Wavefunction.

Chobani's 'natural vs scientific' strategy of promoting foodstuffs isn't new; bloggers have called out Finagle a Bagel on its 'Bakers, not scientists' campaign (http://go.nature. com/jS5PTU), and Whirlpool on their 'Don't drink from the Periodic Table' ads (http:// go.nature.com/6fuqRz). Paul Bracher's 'chemophobia' archive (http://go.nature. com/Lwhove) at ChemBark offers a compilation of examples where science and chemicals are demonized by advertisers.

These discussions highlight that such marketing strategies not only cater to, but also inform the public's paranoia surrounding the term 'chemical'. In his piece (http://go.nature.com/P6e9Tb) on The Blog, Joe Schwarcz addresses the confusion of 'chemicals' and 'dangerous chemicals'. Science education - and communication at all levels has never been more important than the present, where terms like 'GMO', 'GIF' and 'chemical' are misunderstood and feared as a result. It's now time for the chemistry community to use these events as learning and teaching opportunities.

Written by Marshall Brennan, who blogs at http://colorblindchemist.wordpress.com/ 